\title{
GIS: a common operational picture for public safety and emergency management
}

\author{
J. Perdikaris \\ School of Engineering, University of Guelph, Canada
}

\begin{abstract}
Not so long ago, monitoring of remote incidents in real time with dozens of camera feeds and sensors linked seamlessly together was something you would expect from a national intelligence or space agency, or from science fiction. However, first responders, such as police and fire, and local city government buildings, public safety agencies, have used state-of-the-art computers and information systems to capture data for emergencies, but this type of emergency management involved multiple pieces that were not connected, and data certainly was not available in real time using a single seamless interface. GIS has long provided an integration platform for meeting the mission of public safety. This includes providing data management, planning and analysis, field enablement and situational awareness. From 9/11 to Hurricane Katrina, to the 2007 fires in California, to the 2010 Winter Olympics and most recently the earthquake in Haiti, and the Gulf of Mexico oil spill, GIS has been a foundation technology linking data and workflows. A historical perspective of GIS and its integration into public safety is provided, including new developments and challenges facing public safety agencies and emergency managers in regards to GIS are discussed. This includes data acquisition by remote sensing and space technology, imagery, digital terrain and elevation models, geographic and data management systems, topographic representation, spatial and temporal variability of data and linking with numerical models. Data collection, processing and analysis have received a great deal of attention, while data validation, error propagation, and analyses of uncertainty, risk and reliability have not been treated as thoroughly. Furthermore, a discussion regarding the future outlook for GIS and its adoption into Public Safety and Emergency Management is also provided.
\end{abstract}

Keywords: GIS, public safety, emergency management and data analysis. 


\section{Introduction}

Emergency management encompasses a wide range of activities; government at all levels (federal, state, provincial and local) has primary responsibility for emergency management. Traditionally, the military has responsibility for threats from foreign governments and under certain circumstances provide aid in response to national emergencies. Emergencies can be classified as being either human induced or natural occurring. Human induced emergencies include those unplanned events or accidents that occur as a result of human activity or developments. These types of emergencies may include epidemics, war, terrorism, chemical spills, utility failures, crashes, urban fires and riots. Naturally occurring events include forest fires, earthquakes, tsunamis, hurricanes, tornadoes, blizzards, freezes, floods and droughts.

Emergency management encompasses five phases: planning, mitigation, preparedness, response and recovery. All phases of emergency management depend on data from a variety of sources. The appropriate data has to be gathered, organized, and displayed logically to determine the size and scope of the emergency. During emergency first responders often need access to detailed information displayed in a logical manner so that they can respond to and take appropriate action. By utilizing a GIS system, all departments and agencies involved can share information through databases on computer-generated maps in one location. Without this capability, emergency workers must gain access to a number of departments and agencies, their unique maps and data; this in turn requires significant effort and time, resulting in emergency responders having to make decisions without adequate information. This could potentially lead to the loss of lives. GIS provides a mechanism to centralize and visually display critical information during an emergency. This paper will describe how geographic information system (GIS) technology plays a critically important role in public safety and emergency management.

\section{Role of GIS in emergency management}

First responders require effective information tools, to evaluate and manage risk and, at the same time, increase their overall capacity for effective response. Complex incidents involving multiple agencies and organizations require the ability to create common operational pictures that are capable of guiding both preparedness and response. To properly prepare for and manage the possible threats a community might face, sound information technologies, as well as diligence to ensure that collaboration produces useful and relevant information, are required (ESRI [1]). To respond to an emergency event requires significant levels of spatial awareness which can be obtained through the use of a GIS. The following sections provide an overview of how GIS can be used in each phase of emergency management process. 


\subsection{Planning}

Emergency management begins with locating and identifying potential hazard areas. Using GIS, officials can pinpoint hazards and begin to evaluate the consequences of potential emergencies or disasters. For example, when hazards such as earthquake faults, fire hazard areas, flood zones and shoreline exposure are viewed with other mapping data such as streets, pipelines, buildings, residential areas, power lines and storage facilities emergency management officials can begin to formulate mitigation, preparedness, response, and possible recovery needs (Johnson [2]). GIS facilitates this process by allowing emergency planners to view the appropriate combinations of spatial data through computergenerated maps. Through this process emergency planners can then determine where to focus mitigation and response efforts and where they will be most needed during an emergency.

\subsection{Mitigation}

As potential emergency situations are identified, mitigation needs can be determined and prioritized. For example, if a municipality wanted to take preventative action in reducing the amount of flood damages on an annual basis, mitigation may include implementing legislation that limits building in a flood zone. Values at risk such as human life, property, habitat and wildlife can be displayed quickly and efficiently through a GIS. Utilizing existing databases linked to geographic features in GIS makes this possible. A GIS can identify the likely path of a flood based on topographic features. This is accomplished through the use of digital terrain (DTM) and digital elevation models (DEMs). For instance, in the 1980s the primary source of topographic information consisted of contour maps. DEMs automatically extract topographic variables including basin geometry, stream networks, slope, aspect and flow direction. Advances in digital mapping have provided essential tools for representing natural features in 3D (Singh and Woolhiser [3]). The impacts of this flood event can then be quickly identified and targeted for protective action through the use of GIS.

In another example, GIS can be used to map the location of an electrical power grid in the event of an explosion or attack that has caused a loss of power could help emergency response planners know which parts of the community may require more help than others and where the system is most vulnerable. The information could also be used in planning the economic impact of a terrorist attack on the same power system.

\subsection{Preparedness}

Preparedness includes those activities that prepare for actual emergencies. GIS can provide answers to questions during an emergency (Johnson [2]). Such as, who needs to be evacuated from their homes during a hurricane or flood? How many police, fire and paramedic units are required and where should they be located during the event? What evacuation routes should be selected before and 
during the event? Who and will people be notified to evacuate if necessary? Will the road networks handle the traffic? What facilities will provide evacuation shelters? What quantity of supplies, bed space, and so forth, will be required at each shelter based on the number of expected evacuees?

GIS can also display real-time monitoring for emergency early warning system. Remote weather stations can provide current weather indexes based on location and surrounding areas. Wind direction, temperature, relative humidity and rainfall can be displayed by the reporting weather stations. Hydrometric monitoring stations including those used to measure reservoir levels at dam sights and river water levels and flows can all be monitored and displayed by location in GIS. New data collection techniques such as remote sensing, satellite and radar received a great deal of attention in the 1980s and 90s and continue to do so. Major advances have been made in radar, satellite and remote sensing technology in recent years. This technology provides synoptic data of meteorological inputs, landuse, vegetation, moisture content in the watershed and an inventory of water bodies. Radar data is being employed for rainfall measurements in contrast to point rainfall measurements using remote weather stations. Next Generation Weather Radar data is being employed to near realtime high resolution precipitation volume and intensity over space and time (Singh and Woolhiser [3]). It is now possible to deliver this type of information and geographic display over the Internet for public information and awareness or the Intranet for organizational information delivery.

\subsection{Response}

Emergency management assists in the mobilization of emergency services and resources to support first responders for complex emergencies. This can include specialist rescue teams, logistical support, public safety, volunteers, nongovernmental organizations (NGOs), and others. The Emergency Operations Center is responsible to support incident management operation needs and maintain continuity of operations for the community (ESRI [4]). GIS can provide one of the primary components for computer-aided dispatch (CAD) systems. Emergency response units based at fixed locations can be selected and routed for emergency response. The closest response units can be selected, routed, and dispatched to an emergency once the location is known. For example, advanced vehicle locating (AVL) can be incorporated to track in real time the location of incoming emergency units. AVL can also assist in determining the closest mobile units to be dispatched to an emergency, as they are located on the map through global positioning system (GPS) transponders (Johnson [2]).

During multiple emergencies, such as flooding, wildfires and mud slides in different locations, a GIS can display the current emergency unit locations and assigned responsibilities to maintain overall situation status and awareness. If the emergency becomes a disaster and emergency response units arrive from outside the local area, they can be added and displayed.

Depending on the emergency, a GIS can provide detailed information before the first units arrive. For example, before an impending flood arrives within an urban area, the speed and magnitude of the flood wave including resulting 
damage can be modelled to determine evacuation zones and containment needs, such as the placement of sand bags.

\subsection{Recovery}

Once an emergency is over recovery efforts begin and they proceed in two phases' short term and long term recovery. The aim of the recovery phase is to restore the affected area to its original state. It differs from the response phase in its focus; recovery efforts are concerned with issues and decisions that must be made after immediate needs are addressed. Short-term recovery is focused on restoring essential services and support. Long-term recovery efforts are concerned with actions that involve rebuilding destroyed property, reemployment, and the repair of critical infrastructure. GIS is integral for recovery by providing a central information repository for assessment of damage and losses that provides identification of damages including the degree of damage and establish priorities for action (triage) (ESRI [5]). GIS can also assess the overall critical infrastructure damages and short term losses and immediate actions required.

GIS has the capability to display through the primary database the overall current damage assessment. Emergency distribution centers' supplies including medical, food, water and clothing can be assigned in appropriate amounts to shelters based on the amount and type of damage in each area. GIS can then display the number of shelters needed and where they should be located for reasonable access. A GIS can display areas where services have been restored in order to quickly reallocate recovery work to priority tasks. Action plans with maps can be printed, outlining work for each specific area. Shelters can update inventory databases allowing the primary command center to consolidate supply orders for all shelters. The immediate recovery efforts can be visually displayed and quickly updated until short term recovery is complete. This is particularly helpful when managing large emergencies or disasters where work is ongoing in different locations.

Prioritization for major restoration investments can be made with the assistance of GIS. As long-term restoration is completed, it can be identified and visually tracked through GIS. As funds are allocated for repairs, accounting information can be recorded and linked to each location. This can often be a complicated process since long-term recovery costs depending on the impact and magnitude of the disaster can amount to 100s of millions, if not billions of dollars. GIS can help ease the burden of such complicated tasks (ESRI [6]).

In short, emergency management programs are developed and implemented through the analysis of information. The majority of information is spatial and can be mapped. Once information is mapped and data is linked to the map, emergency management planning can begin. Once life, property, and environmental values are combined with hazards, emergency management personnel can begin to formulate mitigation, preparedness, response, and recovery program needs. GIS allows emergency management needs to be identified prior to an incident. Incidents or events can be modelled and displayed in GIS before they occur. Allowing emergency planners to assess the 
consequence of a potential disaster and plan mitigation and response strategies accordingly, thereby reducing the overall vulnerability of the impacted community.

\section{Database management}

For managing and processing large quantities of data GIS becomes an indispensible tool. Integration of GIS with numerical models accomplishes a number of significant functions: design, calibration, scenario simulation, evaluation and comparison of results. For example, in watershed hydrology GIS allows us to subdivide a watershed into several homogeneous subareas in the horizontal and vertical domain. Depending on the type of application, flood forecasting or drought forecasting, requiring hydrologic parameterization or categorization many combinations of spatial overlays can be performed. With GIS it is possible to delineate the impact of a flood, simulate groundwater contamination from point source and non-point pollution and identify areas impacted by drought and low water conditions. GIS enhances the ability to incorporate spatial details beyond the capabilities of numerical models with much better resolution using topography and landuse information derived from aerial imagery, digital terrain and elevation models.

\section{Challenges}

As methods and models of GIS analysis become more sophisticated, the quality of data increases in importance. Many data sets undergo temporal adjustments, which add uncertainty to the analysis. For example, using one- or two-day-old data in flood forecasting at the time of the crisis would lead to a faulty conclusion. The same is true of other disasters where geopolitical or natural conditions change from moment to moment. As a result, we must be able to analyze and incorporate such temporal uncertainty in the analysis and forecast that we make (ESRI [1]).

We must be able to quantify the uncertainty in the data (and the analysis) and express this in a satisfactory mode. A major problem exists in how we report uncertainty in GIS. For example, what significance do we place on the lines on various maps and diagrams? How do we address this issue? A case in point would be designing a hazard map for flooding on an alluvial fan where there is no defined channel and the flood has different probabilities of spreading in various directions. Another major problem is the propagation of uncertainty through the data set as we combine several sets of data of different levels of confidence and even potentially different types (ESRI [1]).

Spatial predictive models perform little to no error analysis. Therefore it is not clear what the model errors are and how they propagate through the different model components and parameters. This is one of major limitations of most spatial predictive models. Therefore, from the user's standpoint, the reliability of a particular model is not clear and therefore, the choice in selecting the 
appropriate model becomes very difficult. Further research on this topic should help to resolve this problem.

\section{Conclusions}

As the emergency management profession continues to evolve, the requirements to deal with complex issues across a variety of disciplines and stakeholders increases. Disaster events are increasing, populations are growing and new threats are beginning to emerge. To meet these demands, specialized tools and technology are required. GIS technology can serve a variety of purposes in supporting the workflows and mission of the emergency management professional. Emergency management requires coordination and collaboration among many stakeholders. The collection of information, analysis of community vulnerabilities, development of mitigation strategies, and overall risk management preparedness is a complex task.

When emergencies occur, key stakeholders must share information on the scope of the event and collaborate on the most effective way to manage the incident and maintain government operations. GIS provides a platform for the common operating picture, where data can be integrated to create a visual description of events. GIS provides a platform for the storage and management of all types of data that can be easily accessed for emergency decision support. The use of GIS is improving how emergency management professionals do their work.

\section{References}

[1] ESRI Inc., Challenges for GIS in Emergency Preparedness and Response. ESRI White Paper. ESRI Inc.: Redlands, pp. 15-20, 2000.

[2] Johnson, R., GIS Technology for Disasters and Emergency Management. ESRI White Paper. ESRI Inc.: Redlands, pp. 3-5, 2000.

[3] Singh, V.P. \& Woolhiser, D.A., Mathematical Modeling of Watershed Hydrology. Journal of Hydrologic Engineering, 7(4), pp. 270-292, 2002.

[4] ESRI Inc., Geographic Information Systems Providing the Platform for Comprehensive Emergency Management. ESRI White Paper. ESRI Inc.: Redlands, pp. 4-8, 2008.

[5] ESRI Inc., Public Safety and Homeland Security Situational Awareness. ESRI White Paper. ESRI Inc.: Redlands, pp. 8-15, 2008.

[6] ESRI Inc., GIS Supporting the Homeland Security Mission. ESRI White Paper. ESRI Inc.: Redlands, pp. 3-6, 2007. 